Journal of Animal and Veterinary Advances 11 (12): 2163-2165, 2012

ISSN: $1680-5593$

(C) Medwell Journals, 2012

\title{
Expression of Etv4 mRNA in Cryptorchidism Mouse Testis and Epididymis
}

\author{
Yang Liu, Guanyu Che, Yixue Sun, Bo Jin, Bin Guo and Xueming Zhang \\ Jilin Province Key Laboratory of Animal Embryo Engineering, \\ The Center for Animal Embryo Engineering of Jilin Province, \\ College of Animal Sciences and Veterinary Medicine, \\ Jilin University, 130062 Changchun, China
}

\begin{abstract}
Previously researchers reported that Etv4, one of the members of Ets transcription factor Etv 5 subfamily, remained at a low level during the normal sexual maturity in testis but a high level in epididymis. Since, the development of spermatogonia and the expression of the regulatory molecules during spermatogenesis could be altered in detrimental environment. In order to examine whether the expression of Etv 4 were changed in male reproductive system when cryptorchidism occurred, expressions of Etv 4 in testis and epididymis were examined in artificial Unilateral Cryptorchidism Mouse Model. Samples were collected on the 3rd, 6th, 12th and 18th days after cryptorchidism surgery, respectively. The effect of cryptorchidism was confirmed histologically. In cryptorchidism testis, Etv $4 \mathrm{mRNA}$ remained steadily at all time points compared with that in the contralateral $(\mathrm{p}>0.05)$. However, it decreased significantly in cryptorchidism epididymis compared with its contrapart on the same time point $(\mathrm{p}<0.01)$ and its decrease continued along with the time especially on day $12(\mathrm{p}<0.05)$ and day $18(\mathrm{p}<0.01)$ compared with day 3 in surgery-lateral epididymis. Collectively, Etv 4 might play an essential role for sperm maturation in epididymis.
\end{abstract}

Key words: Etv4, cryptorchidism, testis, epididymis, mouse, China

\section{INTRODUCTION}

Maintenance of spermatogenesis relies on the selfrenewal and differentiation of Spermatogonial Stem Cells (SSCs) (Nakagawa et al., 2010; Wu et al., 2011). It has been suggested recently that Ets transcription factor Etv 5 plays an important role during the complicate regulation process of SSCs self-renewal and differentiation (Schlesser et al., 2008).

There are three members (Etv1, Etv4 and Etv5) in Etv5 subfamily of Ets family which all share a conserved DNA domain and participate in variety of cell development, differentiation, growth and transformation processes (Oikawa and Yamada, 2003). Previously, researchers have briefly reported that the expressions of Etv5 and Etv1 in normal and damaged mouse testis, respectively (Zhang et al., 2010). However whether the expression pattern of Etv 4 were changed under detrimental circumstance (cryptorchidism) and played possible roles in SSCs development or sperm maturation is still unclear.

It's well known that cryptorchidism is a common disorder of the male reproductive system which affects approximately 2-4\% of male infants (Ashley et al., 2010 ; Xian et al., 2012). This birth defect leads to the failure of the testes to descend into the scrotum correlated with histological changes of the seminiferous epithelia (Hou et al., 2010). Cryptorchidism is an isolated anomaly but a multifactorial disease involving multiple genes (Xian et al., 2012). In this study, researchers further investigated the expression pattern of Ets transcription factor Etv 4 in mouse male reproductive system and discussed its possible function on the development of spermatogonia and the maturation of sperm by using Unilateral Cryptorchidism Mouse Model.

\section{MATERIALS AND METHODS}

Animals and chemicals: Male Kunming White mice (7-8 weeks old, weight 30-35 g) were provided by the Experimental Animal Center of Jilin University (Certification No. scxk-Ji-2007-0003). All experiment mice were performed in accordance with the principles and procedures of Animal Ethics Committee of Jilin University. Pentobarbital sodium was obtained from Sigma Chemicals (St. Louis MO, USA). All other reagents used for molecular biology were purchased from TaKaRa (Dalian, China).

Corresponding Author: Xueming Zhang, College of Animal Sciences and Veterinary Medicine, Jilin University, 5333 Xi-an Road, 130062 Changchun, China 
Unilateral Cryptorchidism Model: Unilateral Cryptorchidism Model was prepared by surgical procedure. Briefly, mice were anesthetized by intraperitoneal injection of pentobarbital sodium $\left(50 \mathrm{mg} \mathrm{kg}^{-1}\right)$. After making a small incision $(\approx 1 \mathrm{~cm})$ in the abdomen, the right testis was pulled into abdomen and its gubernaculum was cut. The displaced testis was further fixed by stitching the fat pad closing the epididymis on the abdominal wall. Contralateral (left) testis served as the control. The displaced testes and their contraparts were collected on days $3,6,12$ and 18 after operation. Tissue samples were stored at $-80^{\circ} \mathrm{C}$ or fixed for further use. Tissue samples were collected from 3 animals $(n=3)$ for each time point.

Haematoxylin-Eosin (HE) staining: Regular HE staining of frozen sections was carried out as researchers described previously.

RT-PCR: As described previously, total RNA from samples was extracted with Trizol. Reverse transcription reactions were carried out with oligo (dT) 18 and the resultant cDNA was frozen at $-20^{\circ} \mathrm{C}$ for later use. First strand cDNA was synthesized from 2 ng of total RNA from testis or epididymis. Reverse transcription was conducted with the 1st Strand cDNA Synthesis kit. Specific PCR Primers of Etv 4 (F: 5'-CACTCCTTCACATC TCCTC-3', R: 5'-AGCATTTGTTGGGTCATC-3', 499 bp) and internal control $\beta$-actin (F: 5'-GATATCGCTGC GCT GGTCGTC-3',R: 5'-ACGCAGCTCATTGTAGAAGGTGT GG-3', 276bp) were synthesized by Shanghai Sangon Biological Engineering Technology and Services Co., Ltd. (Shanghai, China). PCR were performed under following conditions: initial denaturation at $94^{\circ} \mathrm{C}$ for $5 \mathrm{~min}, 94^{\circ} \mathrm{C}$ denaturation for $30 \mathrm{sec}$, annealing $30 \mathrm{sec}$ (Etv4 at $55^{\circ} \mathrm{C}, \beta$-actin at $60^{\circ} \mathrm{C}$ ), extension at $72^{\circ} \mathrm{C}$ for $1 \mathrm{~min}$ and amplification ( 28 cycles for Etv 4 and 20 cycles for $\beta$-actin). Electrophoresis of PCR products were then carried out with $1.5 \%$ agarose gel. The target fragments were recovered and connected with the pGEM-T vector for transformation, screening and sequencing.

Statistical analysis: Semi-quantification was done as before. Briefly, electrophoresis of Etv 4, $\beta$-actin PCR products and DNA marker (DL-2000) were photographed with UV gel imaging system. Grayscale analysis was carried out in triplicate with Gel-Pro Analyzer 4.0 Software. The mean values of Etv $4 / \beta$-actin in same tissue on same time point were used as the expression level of Etv4. Statistical analysis was performed by one-way ANOVA using SPSS 13.0 and the difference was considered significant when $\mathrm{p}<0.05$.

\section{RESULTS AND DISCUSSION}

Histological examination of Cryptorchidism Model: Morphological observation showed that cryptorchidism testes and epididymides withered obviously along time going after surgery, compared with their contraparts. HE staining showed that evident morphological changes occurred in the seminiferous epithelia on day 12 and 18 . The seminiferous epithelia became thinner and thinner, some of them disappeared completely while the interstitial tissue became denser and denser.

Expression of Etv4 in cryptorchidism testis: The electrophoresis of the PCR products of Etv 4 and $\beta$-actin in cryptorchidism testes and their contraparts was shown in Fig. 1a. The semi-quantitative analysis was shown in Fig. 1b. Etv4 mRNA expressed steadily after surgery and no significant difference was observed actually $(\mathrm{p}>0.05)$.

Expression of Etv4 in cryptorchidism epididymis: The electrophoresis of the PCR products in cryptorchidism epididymis and their contraparts was shown in Fig. 2a. Etv 4 mRNA band was obvious weak on day 12 and 18 in cryptorchidism epididymis (Fig. 2a). The semi-quantitative analysis shown that Etv $4 \mathrm{mRNA}$ decreased significantly on all time points compared with their contraparts on the same time (Fig. 2b). Furthermore, Etv4 decreased significantly on day $12(\mathrm{p}<0.05)$ and $18(\mathrm{p}<0.01)$ compared with that of day 3 in surgery side (Fig. 2b).

Ets transcription factor family is widely involved in cells development, differentiation, growth and transformation process and has important functions in many physiological and pathological processes (Davidson et al., 2003; Oikawa and Yamad, 2003; Lettice et al., 2012). Recently, Ets factor Etv 5 was demonstrated to play an essential role in the

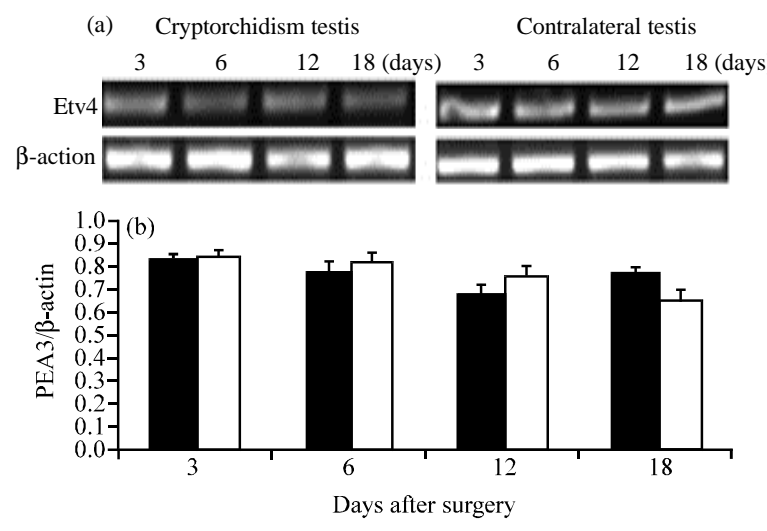

Fig 1: Expression of Etv in cryptorchidism mouse testis; a) Electrophoresis of PCR products; b) semiquantitative analysis (black: cryptorchidism, white: contralateral) 

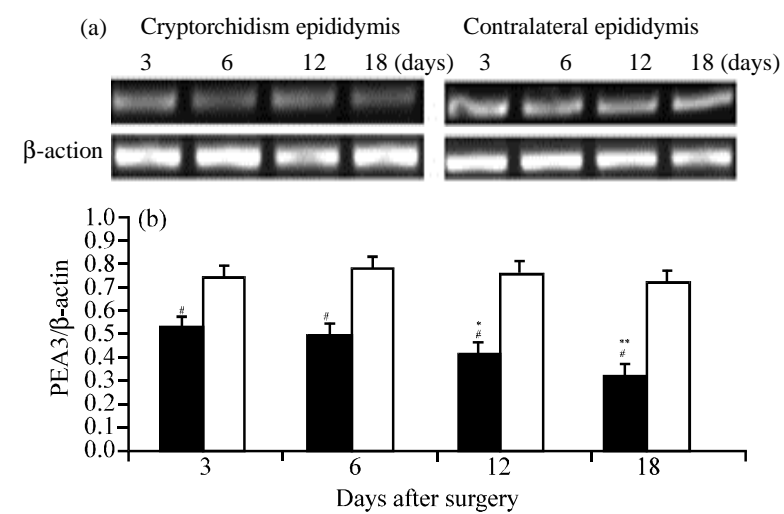

Fig 2: Expression of Etv4 in cryptorchidism mouse epididymis; a) electrophoresis of PCR products; b) semi-quantitative analysis (black: cryptorchidism white: contralateral, ${ }^{*} \mathrm{p}<0.01$ vs. Contralateral at the same time. ${ }^{*} \mathrm{p}<0.01$ vs. cryptorchidism on day 3 , respectively

transcriptional regulation of SSCs niches (Schlesser et al., 2008). However whether its peer members (Etv1 and Etv4) were expressed in male reproductive system and played roles in spermatogenesis is still unknown. Previously, researchers briefly reported the expressions of Etv1 remaining at relatively high level but Etv 4 at very low level in normal testis (Zhang et al., 2010) and Etv4 expression pattern was changed in busulfan-treated mouse testis and epididymis. This study was designed to further check Etv 4 expression under cryptorchidism circumstance, the most common disorder in male reproductive system (Xian et al., 2012).

Generally, testes are situated in scrotum for complete function at testicular temperature. The unilateral cryptorchidism has been widely used as an experimental model for studies of histopathologic changes and molecular genetics of cryptorchidism (Hou et al., 2010; Xian et al., 2012). In this study, morphological analysis indicated that the atrophy of testis and the depletion of spermatogenic cells were similar to that in rat (Tena-Sempere et al., 1999; Hou et al., 2010; Xian et al., 2012) which implied researchers produced the mouse model successfully. Interestingly, expression of Etv 4 in cryptorchidism testis revealed no significant changes but a dramatic decline in epididymis. The results suggest that Etv 4 expression is not affected by higher temperature in testis but indeed affected in epididymis. This is consistent with the former report that the expression of Etv4 in epididymis is involved in the process of sperm maturation.

\section{CONCLUSION}

The Unilateral Cryptorchidism Mouse Model was established successfully. Etv4 expression is not affected by higher temperature in testis but affected in epididymis which implies that it is involved in the process of sperm maturation.

\section{REFERENCES}

Ashley, R.A., J.S. Barthold and T.F. Kolon, 2010. Cryptorchidism: Pathogenesis, Diagnosis, Treatment and Prognosis. Urologic Clin. North Am., 37: 183-193.

Davidson, B., I. Goldberg, W.H. Gotlieb, J. Kopolovic, G. Ben-Baruch and R. Reich, 2003. ETV4 is the second Ets family transcription factor involved in tumor progression in ovarian carcinoma. Clin. Cancer Res., 9: 1412-1419.

Hou, W., J. Hu, Y. Li, J. Zhao and Z. Li et al., 2010. Altered expression of NDRG2 in the testes of experimental rat model of cryptorchidism. Urology, 75: 985-991.

Lettice, L.A., I. Williamson, J.H. Wiltshire, S. Peluso and P.S. Devenney et al., 2012. Opposing functions of the ETS factor family define Shh spatial expression in limb buds and underlie polydactyly. Dev. Cell., 22: 459-467.

Nakagawa, T., M. Sharma, Y. Nabeshima, R.E. Braun and S. Yoshida, 2010. Functional hierarchy and reversibility within the murine spermatogenic stem cell compartment. Science, 328: 62-67.

Oikawa. T. and T. Yamada, 2003. Molecular biology of the Ets family of transcription factors. Genetics, 303: 11-34.

Schlesser, H.N., L. Simon, M.C. Hofmann, K.M. Murphy, T. Murphy, R.A. Hess and P.S. Cooke, 2008. Effects of ETV5 (ets variant gene 5) on testis and body growth, time course of spermatogonial stem cell loss and fertility in mice. Biol. Reprod., 78: 483-489.

Tena-Sempere, M., J. Kero, A. Rannikko and I. Huhtaniemi, 1999. Experimental cryptorchidism induces a change in the pattern of expression of $\mathrm{LH}$ receptor mRNA in rat testis after selective Leydig cell destruction by ethylene dimethane sulfonate. J. Endocrinol., 161: 131-141.

Wu, J.J., T.J. Hu, B. Guo, Z.P. Yue, Z.T. Yang and X.M. Zhang, 2011. Cryopreservation of adult bovine testicular tissue for spermatogonia enrichment. Cryo Lett., 32: 402-409.

Xian, H., Y. Xian, C.Y. Jiang, X. Nie and X.D. Wang et al., 2012. Decreased expression of â-nerve growth factor correlated with histological changes in a cryptorchidism rat model. Chin. Med. J., 125: 713-716.

Zhang, X., L. Si, Z. Yue, Y. Liu, B. Guo, Z. Li and D. Li, 2010. Expression of Ets transcription factors Etv4, Etv1 in mouse testis. Biol. Reprod., Vol. 83. 